\title{
Determinants of subjective contour: Bourdon illusions and "unbending" effects
}

\author{
PETER WENDEROTH, GERARD CRISS, and RICK VAN DER ZWAN \\ University of Sydney, Sydney, New South Wales, Australia
}

\begin{abstract}
Wenderoth and O'Connor (1987b) reported that, although matches to the straight edge of two triangles placed apex to apex revealed an apparent bending in the direction of the chevron formed by the hypotenuse pair (the Bourdon effect), no perceptual unbending of the bent chevron occurred. Using subjective contour figures, Walker and Shank (1988b) found large and approximately equal bending and unbending effects, consistent with two theories that they proposed. In Experiment 1, using adjustable chevron matching and subjective contours, we found that Bourdon effects, equivalent in magnitude to those reported by Walker and Shank, were 4-5 times larger than unbending effects. In Experiment 2, we used a variation of Walker and Shank's measurement technique, in which subjects selected a matching angle from a graded series. We obtained Bourdon effects similar to those in Experiment 1, but much larger unbending effects. Nevertheless, Bourdon effects were significantly larger than unbending effects in one set of data; and in another, Bourdon test means were larger than unbending test means. In both data sets, there was a large and significant pretest bending effect, which enhanced the magnitude of unbending test minus pretest scores. These results were consistent with our theory but not the theories of Walker and Shank. The variance of unbending test matches, 3-4 times that of Bourdon test matches, reflected the task difficulty. We propose that subjective obtuse angle contraction that exceeds real obtuse angle contraction explains the fact that unbending effects are larger in subjective than in real contours.
\end{abstract}

If two identical solid scalene right-angle triangles are placed apex to apex so that the long sides opposite each hypotenuse are aligned (as in Figure 1A), the straight edge so formed appears bent in the direction of the opposite sides. In measuring this illusion, which is termed the Bourdon effect (Figure 1A), Wenderoth and O'Connor (1987b) reported that the truly straight edge of this solid figure appeared bent, but that the bent edge was perceived more or less veridically. However, Walker and Shank (1987, 1988a, 1988b) obtained data to indicate large and significant illusory "unbending" of the truly bent edge, especially when the illusory figure was formed in subjective contours (Figure 1D). For reasons set out below, we aimed to reinvestigate this result.

To measure the effect, Wenderoth, O'Connor, and Johnson (1986a) and Wenderoth and O'Connor (1987a, 1987b) had subjects set a variable chevron, represented by the straight line in Figure 1A, to match the apparent bend. When the truly straight edge of the solid Bourdon figure was oriented $22.5^{\circ}$ clockwise and each vertex an-

This research was supported by Australian Research Council Grant A78831871 to the first author. We thank Senior Systems Analyst John Holden for assistance with all aspects of computing. The experimental program was based on one originally written by Tony $O^{\prime}$ Connor. Two unknown referees were extremely helpful in commenting on earlier versions of the manuscript; and Christopher Tyler suggested using subjective contour displays formed in concentric circles. Correspondence should be addressed to Peter Wenderoth, Department of Psychology, University of Sydney, Sydney, New South Wales, Australia 2006. gle was $12.5^{\circ}$, conditions that maximize the effect (Rozvany \& Day, 1980), Wenderoth and his colleagues reported illusions between $2^{\circ}$ and $4^{\circ}$; that is, the matching chevron was set at an angle of $176^{\circ}-178^{\circ}\left(180^{\circ}=\right.$ straight, physical equality).

In one experiment, Wenderoth and O'Connor (1987b) presented Bourdon type figures that actually did have bent edges; one of these figures was that shown in Figure 1B, in which the nonmatched edge was straight. Thus, the subject's task was to match the bent left-hand edge using the adjustable chevron. In all of their experiments, Wenderoth and his colleagues (Wenderoth \& O'Connor, 1987a, 1987b; Wenderoth et al., 1986a) defined the illusion as the difference between a pretest control measure and the test measure. In the pretest, the variable chevron was matched to thin lines that defined the test edge, but in the absence of the triangular shapes. That is, the subjects first matched an angle to itself, although the lengths of the two angle arms were different to avoid global strategies such as making parallelograms. Wenderoth and O'Connor (1987b) reported that significant illusions occurred only when the matched edge was straight, a finding that contributed to their conclusion that the Bourdon illusion depends on acuity for straightness and may involve the outputs of separate neural channels coding orientation, position, and collinearity (see below).

In stark contrast to these findings, Walker and Shank (1987, 1988a, 1988b) have reported large and significant "unbending" effects. That is, in displays such as Figures $1 \mathrm{~A}$ and $1 \mathrm{~B}$, they found that both the straight edge 


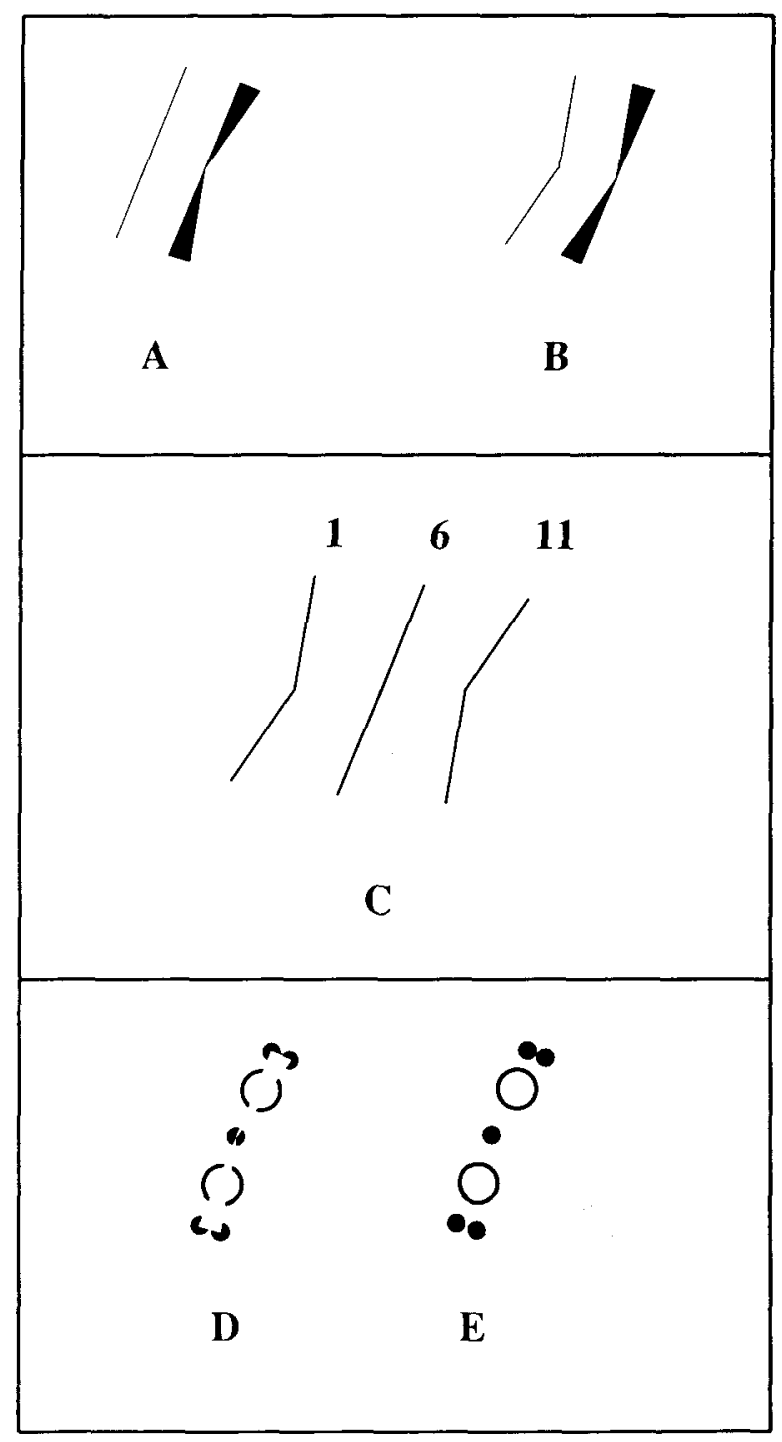

Figure 1. (A) The Bourdon illusion: The straight edge formed by mirror reflecting the filled triangle about its apex appears bent and can be matched by a variable chevron represented by the thin straight line. (B) The "unbending" display used by Wenderoth and O'Connor (1987b). (C) Examples of comparison stimuli used by Walker and Shank (1987, 1988a, 1988b). (D) A Bourdon figure in subjective contours, after Walker and Shank (1987). (E) Control stimulus, after Walker and Shank (1987).

and the truly bent edge underwent illusory displacement. They offered not only a cognitive confusion model, but also a feature detection model to account for the fact that all figure edges appear displaced toward the center of the figure, a bending illusion of the straight edge and an unbending (i.e., straightening) illusion of the bent edge.

A major concern in all of the Walker and Shank (1987, $1988 \mathrm{a}, 1988 \mathrm{~b}$ ) experiments has been to compare the magnitudes of Bourdon and unbending effects in figures composed of real versus subjective contours. Before we address this issue of contour type, let us summarize the current theories of the Bourdon illusion and the data that bear upon those theories. Walker and Shank (1988b) proposed two theories, which, as they noted, made predictions that were "essentially indistinguishable in the present study" (p. 483). Their feature detector theory, similar to that of Carpenter and Blakemore (1973) and O'Toole and Wenderoth (1977), proposed that a line induces a "Mexican hat" distribution of excitation and flanking inhibition in a population of cortical orientation selective neurons. Two lines at nearby orientations will usually repel each other perceptually (the tilt illusion), because linear summation of two such overlapping distributions will result in net excitatory peaks that are skewed apart (see also Wenderoth, O'Connor, \& Johnson, 1986b). A solid angle such as one of those in Figure 1A, however, will induce neural distributions with additional excitation between the peaks of firing (induced by the contrast differences at the angle edges) because of the solid contour between those edges (Walker \& Shank, 1988b, Figure 2D). As a result, summation of excitation and inhibition will result in peaks of firing skewed toward each other. This, they argue, would account for bending and unbending effects. Walker and Shank's second model, a cognitive confusion model, attributes both the bending and unbending effects to "the confusion of the test and induction contours"' (Walker \& Shank, 1988b, p. 476).

It can be noted that both of these theories would have to predict that similar illusions would occur regardless of whether the stimulus used was a double-angle display, such as that in Figure 1A, or a single-angle display, containing just the upper or the lower triangle of Figure 1A. However, Wenderoth et al. (1986a) compared the effects for filled and line versions of the Bourdon figure. They found, first, that upper and lower single-angle matches showed repulsion effects (tilt illusions or angle expansions, called + ve) for outline angles but attraction effects (angle contractions, called -ve) for solid angles. Second, errors in chevron matches were reduced to near zero for outline double-angle displays, whereas in solid doubleangle displays, errors were significantly more negative than the sum of the upper and lower single-angle matches.

Nothing in either of the Walker and Shank (1987, $1988 \mathrm{a}, 1988 \mathrm{~b})$ theories could explain these results, although, since the experiments above preceded those of Walker and Shank, we did not make this point at the time. We proposed the theory that there are separate neural channels coding orientation, collinearity, and position, a suggestion made elsewhere in the context of the tilt aftereffect by Harris and Calvert (1985).

In these terms, outline figures provide opposing cues to orientation on the one hand and straightness and collinearity on the other, so that net matching errors are small. Solid figures provide less salient cues to straightness and collinearity, because the matched edge is not physically isolated from the rest of the figure. The absence of these cues results in large errors, but only when the matched edge is physically straight; observers can make absolute judgments about straightness but not about degrees of nonstraightness. Wenderoth and O'Connor (1987b) found, as predicted, that significant negative il- 
lusions occurred with solid angle Bourdon figures only when the test edge was straight, and that outline illusions were significantly more positive when the test edge was physically bent.

Finally, Wenderoth and O'Connor (1987b) noted that, like many other illusions such as the Zöllner and Poggendorff effects, the tilt illusion is larger when the test edge is oblique than when it is vertical or horizontal, presumably because of anisotropies in the number and tuning width of orientation channels at different orientations (Howard, 1982). Nevertheless, these illusions are significant, albeit smaller, when the test lines are vertical or horizontal. If solid angle illusions are pure acuity effects, it was argued, they should occur only around more oblique orientations where orientation acuity is weak, and they should be eradicated (rather than merely reduced) around the main axes of space. This was shown to be the case (Wenderoth \& O'Connor, 1987b, Experiments 1, 3, and 4) and, in addition, manipulations that decrease acuity for parallelism (e.g., increasing separation of matched edges, decreasing length of test edges) were demonstrated to have the effect of increasing solid angle illusions, whereas they either decreased or had no effect on outline illusions (Wenderoth \& O'Connor, 1987b, Experiments 5 and 6).

In the light of these results, Wenderoth and $O^{\prime}$ Connor (1987b) concluded that outline and solid double-angle (Bourdon) illusions have different explanations. The outline illusion involves a pair of tilt illusions (angle expansion), probably due to lateral inhibitory processes (see, e.g., Carpenter \& Blakemore, 1973; Wenderoth et al., 1986b), offset by cues to collinearity. The solid illusion involves merely a failure adequately to discriminate the orientation of the judged edge from the orientations of the angles' bisectors (a shorthand way of expressing the proposed neural compromise between orientation, position, and collinearity signals).

Given this background of previous research and theory, let us now consider the experiments of Walker and Shank (1987, 1988a, 1988b), who examined Bourdon effects not only using real contours such as those in Figure 1A, but also using subjective contour displays such as that in Figure 1D. They found that both bending and unbending effects were considerably larger in subjective contour figures. For example, Walker and Shank (1988b) reported bending and unbending effects of about $4^{\circ}$ and $1.5^{\circ}$ for Figure $1 \mathrm{~A}$ stimuli but effects of about $9^{\circ}$ and $5.5^{\circ}$ for Figure 1D stimuli. In their earliest experiments, Walker and Shank (1987) found even larger subjective contour effects $-10^{\circ}$ and $9^{\circ}$ bending and unbending effects, respectively-but, as they themselves noted, there were possible anchoring artifacts in their measurement procedure.

To circumvent this problem, Walker and Shank (1987) changed the measurement procedure. They presented subjects with five chevrons, two bent in the direction of Stimulus 1 in Figure 1C, one straight, and two bent in the direction of Stimulus 11 in Figure 1C. The internal angles of the nonstraight stimuli were $155^{\circ}$ and $130^{\circ}$. These stimuli were named to the subjects as "20L,"
"10L," "0," "10R," and "20R." The subjects were required to pick a scale value, interpolating as necessary. According to Walker and Shank (1987, p. 20) these comparison stimuli " would allow the measurement of repulsion as well as attraction, or assimilation, of the bent edges by the colinear edges, thereby eliminating or reducing the possible response biases ... in the earlier experiments."

Using this method, Walker and Shank obtained subjective contour bending and unbending effects of about $8.5^{\circ}$ and $7.5^{\circ}(1987), 6^{\circ}$ and $7^{\circ}(1988 \mathrm{a})$, and $9^{\circ}$ and $5.5^{\circ}$ (1988b), respectively. These results suggest that bending and unbending effects are approximately equivalent in subjective contour figures, a prediction made by both Walker and Shank's feature detector model and their cognitive confusion model. With ordinary filled contours (Figure 1A), however, Walker and Shank always obtained considerably smaller unbending than bending effects, which, as they acknowledge, does not sit well with their theories (Walker \& Shank, 1988b, p. 479).

Our aim is to reexamine bending and unbending effects in subjective contour figures. Although Wenderoth and O'Connor (1987b) reported no unbending effect in figures such as Figure 1B, examination of their data (Figure 3, p. 48) shows that in the relevant condition (D), a small though insignificant effect in the unbending direction did occur, of the order of $0.3^{\circ}$ (representing the illusion in one of the two chevron arms, or half the total illusion). In the same data, the Bourdon or bending effect was about $1.3^{\circ}$, so that even though the unbending effect was not significant, it was of the order of $25 \%$ of the Bourdon effect. Walker and Shank $(1987,1988 \mathrm{~b})$ have typically obtained an unbending effect in nonsubjective, but solid, figures of about $40 \%$.

On the one hand, we were partly concerned that we might have made a Type II error, thus failing to detect an unbending effect. We were concerned also that we had suggested that unbending effects do not occur because subjects have low acuity for differences between bent chevrons and we had predicted no unbending effect. If it were true that bending and unbending effects are almost equivalent when subjective contour figures are used, then our analysis of the Bourdon effect would be severely compromised. On the other hand, we suspected that Walker and Shank $(1987,1988 \mathrm{a}, 1988 \mathrm{~b})$ may have overestimated the magnitude of unbending effects, for the following reasons:

First, we were not convinced that the modified method of measurement adopted by Walker and Shank (1987, $1988 \mathrm{a}, 1988 \mathrm{~b}$ ) was satisfactory, because the stimulus in the matching series that physically equalled the bent edge was still not centered in the series. This could have resulted in regression to the mean of the series and hence in a constant "unbending effect" due purely to response bias. This view is given credence by the fact that when Walker and Shank (1988b) introduced a control figure (similar to Figure 1E above) and asked subjects to match the virtual bent line passing through the circles, they obtained an unbending effect (albeit nonsignificant) of $1.53^{\circ}$ in a stimulus that "was not expected to show any significant displacement"' (p. 479). 
Second, even if this control effect was not significant, it would have been prudent to subtract each subject's control match from each test match. The bending control match was -0.43 . Hence, whereas Walker and Shank (1988b) reported bending and unbending effects for solid real contours of about $6^{\circ}$ and $2.75^{\circ}$, test minus pretest scores would have given bending and unbending effects of $6.4^{\circ}$ and $1.25^{\circ}$, respectively. That is, without subtracting pretest controls, the unbending effect is $46 \%$ of the bending effect; after pretest subtraction, this reduces to $20 \%$, which is much closer to the value estimated from Wenderoth and O'Connor's (1987b) data (above).

Third, we were concerned about the nature of the subjective contour figures. As can be seen in Figure 1D, there are so many background real contours in the subjective figure that it is difficult perceptually to isolate the edge to be judged. We wondered whether it might not have been more judicious to use subjective contours produced by line ends, of the kind used more frequently in current research, both psychophysical (Vogels \& Orban, 1987; Paradiso, Shimojo, \& Nakayama, 1989) and neurophysiological (von der Heydt \& Peterhans, 1989).

Aside from these methodological issues, our main aim was to ascertain whether Bourdon and unbending effects are or are not equivalent in magnitude. Our theory (Wenderoth \& O'Connor, 1987a, 1987b; Wenderoth et al., 1986a) predicts smaller or nonexistent unbending effects; Walker and Shank's $(1987,1988 \mathrm{a}, 1988 \mathrm{~b})$ two theories predict equal and opposite Bourdon and unbending effects.

In Experiment 1, using stimuli such as those shown in Figure 2, we chose to reinvestigate the relative magnitudes of bending and unbending effects. To measure the Bourdon or bending effect, we first had subjects match a real line chevron to a subjective rectangle (Figure 2A). Then the subjects matched the same chevron to a subjective Bourdon edge (Figure 2B), and the bending effect was defined as the test minus pretest difference. Bends that produced right-facing obtuse angles (as Stimulus 11 in Figure 1C) were called negative, so that a test minus pretest Bourdon effect would be negative. Figures $2 \mathrm{C}$ and 2D show the pretest control and test unbending displays, in which a test minus pretest unbending effect would be positive.

Subjective contour displays were used because with these Walker and Shank (1987, 1988a, 1988b) obtained the largest bending and unbending effects, and we wanted to maximize our chances of obtaining unbending effects. We also used two densities of horizontal lines to produce the subjective contours so that the gaps between the lines were either $0.2^{\circ}$ or $0.1^{\circ}$, in case line density affected the "salience" of the subjective contours and perhaps the illusions induced. We were aware of the fact that the stimuli we used (Figure 2) were not "pure" subjective contour displays, in that Fourier analysis of these stimuli (or severe blurring) would reveal real contours. This could have been avoided by including other horizontal lines within the white segments but out of phase with the background lines. This was not done for two reasons. First, the extra lines caused screen flicker. Second, Walker and Shank (1988b)
A. BOURDON CONTROL
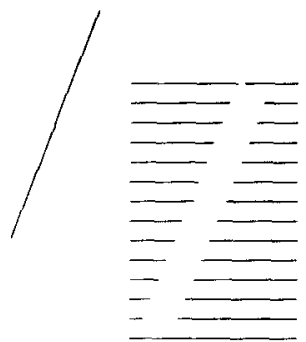

C: UNBENDING CONTROL

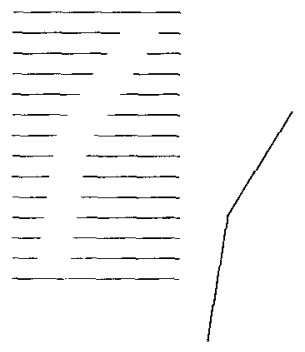

Figure 2. Subjective Bourdon pretest (A) and test (B) figures and unbending pretest (C) and test (D) figures used in Experiment 1.

obtained much larger effects with solid than with outline subjective figures.

\section{EXPERIMENT 1}

\section{Method}

Apparatus. Stimuli were generated on the flat screen of a Kikusui COS1611 monitor (P31 phosphor) using a Hewlett-Packard 1350A Graphics Translator linked to a PDP-11/20 computer. The subject sat in a dark, windowless, sound-attenuating cubicle and viewed the screen from a padded chinrest $57 \mathrm{~cm}$ from the screen, so that $1 \mathrm{~cm}$ subtended $1^{\circ}$ of visual angle. Communication between subject and experimenter, the latter outside the cubicle, occurred via intercom. Using three microswitches, the subject could bend the matching chevron left or right (outer switches) or indicate a satisfactory match, cause the data to be stored, and end the trial (center switch).

Stimuli. The stimulus displays are schematically represented in Figure 2. The overall size of the line-filled rectangle in which the test figures were embedded was $2.8^{\circ}$ high and $1.8^{\circ}$ wide. Either 14 or 28 horizontal lines filled this area, so the lines were separated by $0.2^{\circ}$ or $0.1^{\circ}$. The straight edges in Figure 2 were oriented $67.5^{\circ}$ (horizontal right $0^{\circ}$, angles measured counterclockwise). In the case of the bent edges, the upper chevron arm was at $55^{\circ}$ and the lower arm was at $80^{\circ}$, so that the internal angles in the Bourdon figures were $12.5^{\circ}$. Each chevron arm in the test figures was $1.3^{\circ}$ long, so that the total length of the matched edge was $2.6^{\circ}$. The matching chevron was $2.5^{\circ}$ long and was positioned $1.6^{\circ}$ orthogonally to the axis of mirror symmetry of the test display.

Partially crossed polaroids were inserted behind the circular viewing mask, which equated the luminance of all lines at $1.8 \mathrm{~cd} / \mathrm{m}^{2}$ 
as measured by a Tektronix $\mathrm{J} 161^{\circ}$ luminance probe, and Michelson contrast, defined as $\left[L_{\max }-L_{\min }\right] / L_{\max }+L_{\min }$ ], was close to 1.0 .

Subjects. Nineteen subjects from an introductory psychology course served as volunteers in return for nominal course credit. All had emmetropic or suitably corrected vision.

Procedure. Each subject received all 32 conditions in a randomized factorial design: 2 effects (bending vs. unbending) $\times 2$ line densities ( 14 vs. 28$) \times 2$ trial types (control and test) $\times 4$ starting positions (matching chevron set $\pm 5^{\circ}$ or $\pm 10^{\circ}$ ).

On arrival, subjects were shown sketches of the stimuli, and it was explained to them that the chevron was always to be matched to the nearest edge of the subjective figure. Three practice trials ensured that the subject knew what was required and was able to complete the task comfortably. Careful bracketing during adjustment was encouraged.

All errors were measured as the deviation of one arm of the chevron from physical equality, in degrees.

\section{Results}

Mean chevron setting errors, together with standard errors, are presented in Table 1 for all eight conditions. Test minus pretest illusion means are given in the last row. In the control and test conditions, positive errors indicate that, relative to physical equality, the upper chevron arm was set too far counterclockwise and the lower arm was set too far clockwise. Negative errors indicate the opposite.

Considering first the Bourdon (bending) effect, it can be seen that the pretest control setting errors to the subjective rectangle (Figure $2 \mathrm{~A}$ ) were very small, $+0.13^{\circ}$ and $+0.17^{\circ}$ in the 14 - and 28-line displays, respectively. For the Bourdon display (Figure 2B) setting errors were in the expected, negative direction, $-3.91^{\circ}$ and $-3.79^{\circ}$ for the two displays. The subtraction of pretest from test measures gave Bourdon illusions of $-4.04^{\circ}$ and $-3.97^{\circ}$. Since these measures refer to just one chevron arm, the full angular effects, $-8.08^{\circ}$ and $-7.94^{\circ}$, are similar to the average of subjective contour Bourdon effects reported by Walker and Shank $(1987,1988 \mathrm{a}, 1988 \mathrm{~b})$ (see introduction) of about $7.3^{\circ}$.

However, the unbending effects we obtained were quite different from those of Walker and Shank $(1987,1988 \mathrm{a}$, $1988 \mathrm{~b}$ ) in a number of respects. First, while Walker and

Table 1

Means and Standard Errors of Bourdon and Unbending Matching Errors, in Degrees

\begin{tabular}{|c|c|c|c|c|}
\hline & \multicolumn{4}{|c|}{ Condition } \\
\hline & \multicolumn{2}{|c|}{14 Lines } & \multicolumn{2}{|c|}{28 Lines } \\
\hline & Bourdon & Unbending & Bourdon & Unbending \\
\hline \multicolumn{5}{|c|}{ Control } \\
\hline Mean & +0.13 & -1.61 & +0.17 & -3.05 \\
\hline Standard error & 0.12 & 0.35 & 0.11 & 0.28 \\
\hline \multicolumn{5}{|c|}{ Test } \\
\hline Mean & -3.91 & -0.67 & -3.79 & -2.16 \\
\hline Standard error & 0.55 & 0.45 & 0.57 & 0.46 \\
\hline \multicolumn{5}{|c|}{ Test Minus Pretest } \\
\hline $\begin{array}{l}\text { Mean } \\
\text { Standard error }\end{array}$ & $\begin{array}{r}-4.04 \\
0.51\end{array}$ & $\begin{array}{r}+0.94 \\
0.28\end{array}$ & $\begin{array}{r}-3.97 \\
0.56\end{array}$ & $\begin{array}{r}+0.89 \\
0.30\end{array}$ \\
\hline
\end{tabular}

Note-All errors are for one arm only.

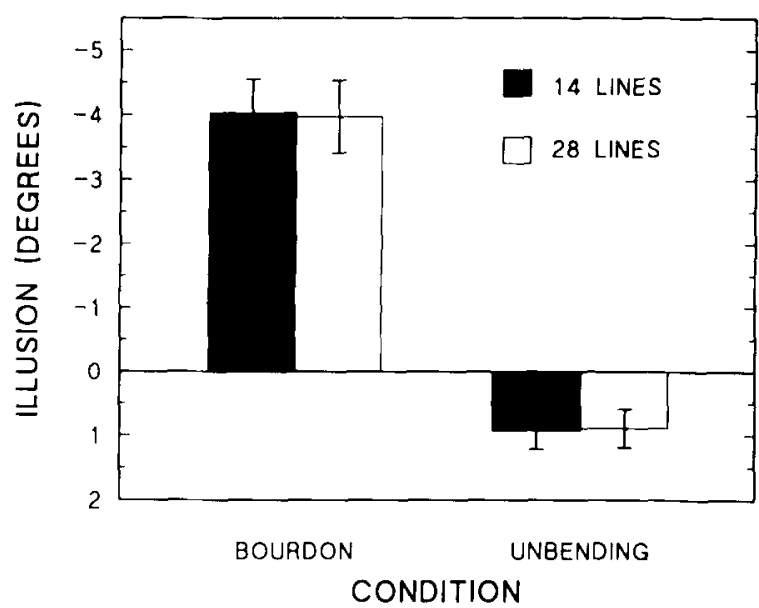

Figure 3. Mean Bourdon (bending) and unbending illusions in 14 and 28-line displays. Error bars show $\pm 1 S E$.

Shank's effects averaged $6^{\circ}-7^{\circ}$, our half effects were $+0.94^{\circ}$ and $+0.89^{\circ}$ (Table 1 ), giving full effects of only $+1.88^{\circ}$ and $+1.78^{\circ}$. It can be noted that these unbending effects constitute $23 \%$ of the Bourdon effect, similar to the percentages we have obtained previously and similar to the percentage Walker and Shank would have obtained had they subtracted pretest control measures from test measures (see introduction). Second, had we used test measures only, as did Walker and Shank, we would have obtained not an unbending but a bending effect; as Table 1 shows, all of the control and test measures for the unbending effects were negative, indicating that the matching chevron was set more bent than the test chevron. The test minus pretest effects were positive because the small unbending effect reduced the control bending effect but did not offset it completely.

The illusion data (Figure 3) were subjected to a repeated measures single-factor analysis of variance with three planned contrasts. That is, although the complete design can be regarded as a $2 \times 2 \times 2 \times 4$ factorial, we were not interested in starting position or pretest means per se but rather in the test minus pretest differences averaged across starting positions.

Bourdon and unbending illusions were significantly different from each other both in the 14-line displays $[F(1,54)=64.49, p<.0005]$ and in the 28-line displays $[F(1,54)=61.52, p<.0005]$, but the difference between the two line-density effects was not significant $[F(1,54)=0.0007, p>0.05]$.

Single mean $t$ tests using the variances associated with each mean showed that both the 14- and 28-line Bourdon effects were different from zero $[t(18)=-7.89$ and -7.08 , respectively, $p<.0005$ in both cases], as were the unbending effects $[t(18)=3.35$ and 2.96 , respectively, $p<.01$ in both cases]. It may be noted that while all 38 measured Bourdon effects were negative, 9 of the unbending measures were also negative-that is, they were in the wrong direction. 


\section{Discussion}

The results of this experiment show that when the chevron matching technique is used to measure Bourdontype illusions, relatively small unbending effects occur in subjective contour figures. These effects were of the order of $20 \%$ of Bourdon (bending) effects. Thus, whereas we did obtain very large Bourdon effects with subjective contours, comparable to those reported by Walker and Shank $(1987,1988 \mathrm{a}, 1988 \mathrm{~b})$, we did not find that unbending effects in these figures were comparable in magnitude to bending effects.

We attribute the differences in results to one or more of the three factors discussed in the introduction: (1) Walker and Shank $(1987,1988 a, 1988 b)$ used a measurement procedure that could have magnified unbending effects via response bias; (2) Walker and Shank did not subtract pretest control measures from test measures; and (3) the subjective contour figures used by Walker and Shank contained many real contours, some of which contained elements at angles different from, but very close to, that of the edge to be judged. In relation to this last point, it is not at all obvious what total effect the complex contours in Figures 1D and 1E would have upon an ensemble of orientation-selective neurons. This concern is reinforced by the fact, mentioned earlier, that a control figure similar to that in Figure 1E but with two small filled circles instead of outline circles gave an unexpected, though nonsignificant, $1.53^{\circ}$ unbending effect; and by the fact, not mentioned before, that in their first study, using their admittedly flawed methodology, Walker and Shank (1987) used the precise control figure shown in Figure 1E and obtained a nonsignificant bending effect of under $1^{\circ}$ but a highly significant unbending effect in excess of $6^{\circ}$. It is difficult to believe that the bias in the matching series alone could produce such a large and significant unbending effect in a control figure lacking any perceptible straight edges.

In discussing some differences between our earlier results and their own, Walker and Shank (1988b) pointed to our chevron matching task as one possible determinant of the discrepant data. However, our "replication" of the large Bourdon effects with subjective contours here seems to argue against any special difficulty associated with the chevron matching method.

Some additional aspects of the Experiment 1 data require discussion. First, as noted earlier, the unbending effects we obtained were actually the test minus pretest differences between two bending effects; the subjective chevron edge in Figures $2 \mathrm{C}$ and $2 \mathrm{D}$ was matched by a smaller obtuse angle in both the test and pretest. One possible explanation is that the figures are complex and there were complex interactions in the orientation domain between the subjective contours at $55^{\circ}$ and $80^{\circ}$ and the real horizontal line segments. This is a problem inherent in the kinds of subjective contours we have used. We could have used lines that were orthogonal to the Bourdon (straight) edge to generate our subjective contours, but these would still have made a $77.5^{\circ}$ angle with the bent subjective chevron. If these angles induced an indirect tilt illusion (an angle-arm attraction effect), as $70^{\circ}-80^{\circ}$ angles often do (see Wenderoth \& Johnstone, 1987; Wenderoth et al., 1986b), this would have produced a spurious bending effect on both bent chevron arms.

Our methodology has been to use test minus pretest differences to attempt to factor out any such interactions that might have occurred. Thus, even if the real lines in our displays affected the perceived orientations of the subjective edges, and even if these lines also affected the perception of the matching chevrons, such interactions would also have occurred in the pretest control conditions. It can be demonstrated readily from the data we obtained that indirect tilt illusions did indeed occur, and that the test minus pretest technique did factor them out.

It is well documented that Zöllner and related tilt illusions induced by tilted lines on a single contour increase in magnitude with inducing line density (see, e.g., Wallace \& Crampin, 1969). Therefore, if tilt induction did occur in Experiment 1, it should have been greater in the 28- than in the 14-line condition. Table 1 shows that this was the case for the unbending conditions. In the pretest controls, the 28-line unbending pretest was more negative than the 14 -line pretest, by $1.44^{\circ}\left(-3.05^{\circ}\right.$ minus $\left.-1.61^{\circ}\right)$. In the test, the 28 -line unbending effect was also more negative than the 14-line effect, by $1.49^{\circ}\left(-2.16^{\circ}\right.$ minus $-0.67^{\circ}$ ). The difference between these two tilt illusions, $0.05^{\circ}$, is clearly not significant. Hence, the attraction tilt effect caused by the interaction between the real horizontal lines and one or both of the subjective unbending edges has been factored out by subtraction. Why is there no such effect in the Bourdon measures? The Bourdon edge is truly straight; hence, any induced tilt will shift both angle arms in the same direction and by the same amount, without affecting perceived straightness.

It might be argued also that we somehow confounded our results by changing the position of the matching chevron from "bending" to "unbending" conditionsthat height in the visual field, for example, is important. However, although elevation is known to affect judgments related to size and depth, there is no evidence that it affects two-dimensional angle judgments, and we preferred at any rate to have the matching angle directly juxtaposed to the edge to be judged. Had we placed the matching chevron directly below the display in all conditions, for example, it would have been nearest the bent edge, but the bent edge would have been between the test and matching edges in the bending condition, a confound we wished to avoid.

A more plausible explanation of the fact that our unbending effects were the difference of two bending illusions is that obtuse angles are underestimated (Carpenter \& Blakemore, 1973) and that they are more underestimated in subjective than in real contours. That is, tilt illusions are larger in subjective contours. If this were so, our subjective bent chevrons would appear more bent than the real matching chevrons when the angles were physically equivalent, which would result in a significant bending effect in the pretest, as we found. This point about tilt effects in subjective contours is taken up further below. 
A further aspect of the data that requires comment is the failure to find any effect of line density on test minus pretest effect magnitudes. Recently, von der Heydt and Peterhans (1989) reported studies on monkey V2 cells, which respond to anomalous contours, and in one experiment they found that some, not all, cells were tuned to contour density. However, even when cells did respond better to contours formed by dense rather than sparse lines, the difference became apparent with densities much less than those in our two conditions. That is, the cells studied by von der Heydt and Peterhans would have given equally strong responses to the subjective contours in both of our conditions. In addition, as noted earlier, our displays were such that they would stimulate V1 cells, which do not respond to anomalous contours (von der Heydt \& Peterhans, 1989). It remains to test Bourdon effects in truly subjective contours and with a variety of line densities-including densities considerably more sparse than those used here.

Given that we were not able to produce computergenerated subjective contours that were unquestionably free of possible unwanted orientation interactions (it is simply not possible with our equipment to produce circular figures), we decided to conduct a second experiment using methods more similar to those of Walker and Shank and using subjective contours formed by lines orthogonal to those contours.

\section{EXPERIMENT 2}

The stimulus displays used in this experiment are shown in Figures 4A (Bourdon control), 4B (unbending control), and $4 \mathrm{C}$ (Bourdon and unbending test). In these displays, it was intended that all the circular contours meet all subjective edges at right angles, precluding any possibility of spurious interactions in the orientation domain. However, we realized during the experiment that this was not true for Figures $4 \mathrm{~A}$ and $4 \mathrm{~B}$, and also that whereas the circular contours were orthogonal to the judged pretest edge in Figure 4A, they were not orthogonal to the unbending pretest edge in Figure 4B. We therefore replicated the experiment, using Figures $4 A$ and $4 \mathrm{C}$ as before, but substituting Figure 4D for 4B; in Figure 4D, the circular contours are orthogonal to the judged edge.

We refer to the original and "replicate" experiments as Experiments 2.1 and 2.2, respectively.

Figure 5 shows the two graded series of 31 angles used by subjects to match the edges to be judged. In both the Bourdon (Figure 5A) and the unbending (Figure 5B) series, the center stimulus was physically equal to the judged edge, and the angles on either side of it were incremented or decremented from it in $1^{\circ}$ steps. Thus, in Figure 5A, the extreme left- and right-hand stimuli have internal angles of $165^{\circ}$ and the center line is straight.

\section{Method}

Apparatus and Stimuli. All stimulus displays were created on an Amiga 2000 computer using Professional Draw software and
A

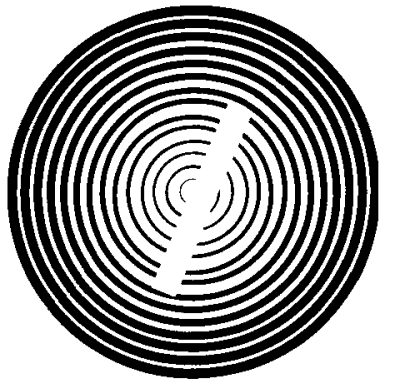

$\mathbf{B}$

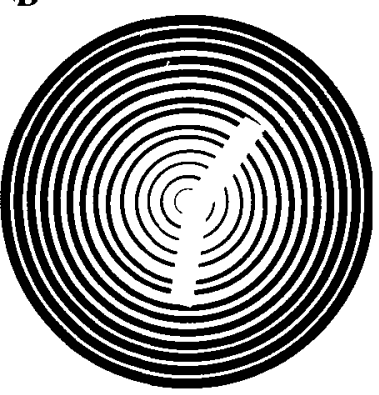

C

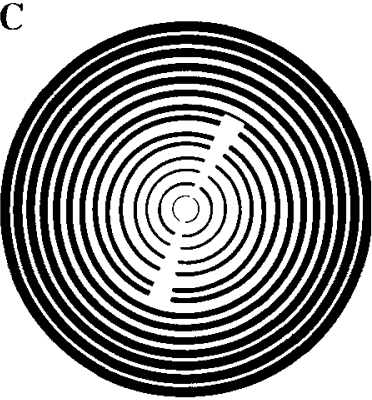

$\mathbf{D}$

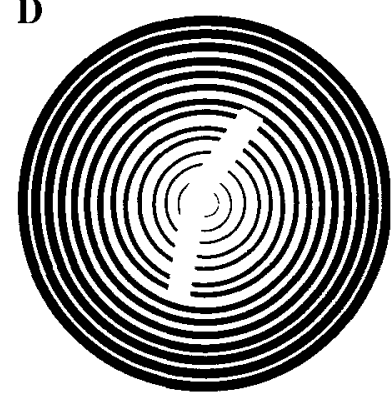

Figure 4. Subjective Bourdon pretest (A), unbending pretest (B), and test stimulus (C), Experiment 2.1. Unbending pretest stimulus, Experiment 2.2 (D).

printed on white paper using Postscript files and an Apple Laserwriter II. All angle arms, both those judged and those used for matching, were $2.54 \mathrm{~cm}$ long. The total diameter of the concentric circular fields was $9.5 \mathrm{~cm}$. The circles increased in width from the center because the displays were created by cloning, and then magnifying, the center circle, a property thought not to be of critical importance except that it was found to increase the salience of the subjective edges. The software used to produce the circles introduced some uneven spacing between them, but this was also deemed not critically important. All angular properties of the subjective contour stimuli were as in Experiment 1.

Subjects. Experiment 2.1, in which Figure 4B was used, was conducted during a semester break so that the population of subjects used in Experiment 1 was not available. The subjects in this part of the experiment were 18 staff members of the Department of Psychology, including graduate students and technical staff, who had no knowledge of visual perception beyond any undergraduate training, and 2 members of the Department of Religious Studies, giving 20 subjects in all. In Experiment 2.2, in which Figure 4D was used instead of Figure 4B, there were 34 subjects, drawn from the same population as those in Experiment 1 .

Procedure. The subjects were first shown one of the two graded series of angles. They were told that the task was to look at various displays and to choose one of the numbered angles as a match to a test angle. A careful warning was given that the true match could come from any position at all in the series and that no bias should be shown toward any particular location in the series. Each subject then made the four judgments in a different random order. The judgments were: (1) to use the Figure 5A series to match the left-hand subjective edge in Figure 4A (Bourdon pretest); (2) to use the same series to match the left-hand edge in Figure $4 \mathrm{C}$ (Bourdon test); (3) to 
A
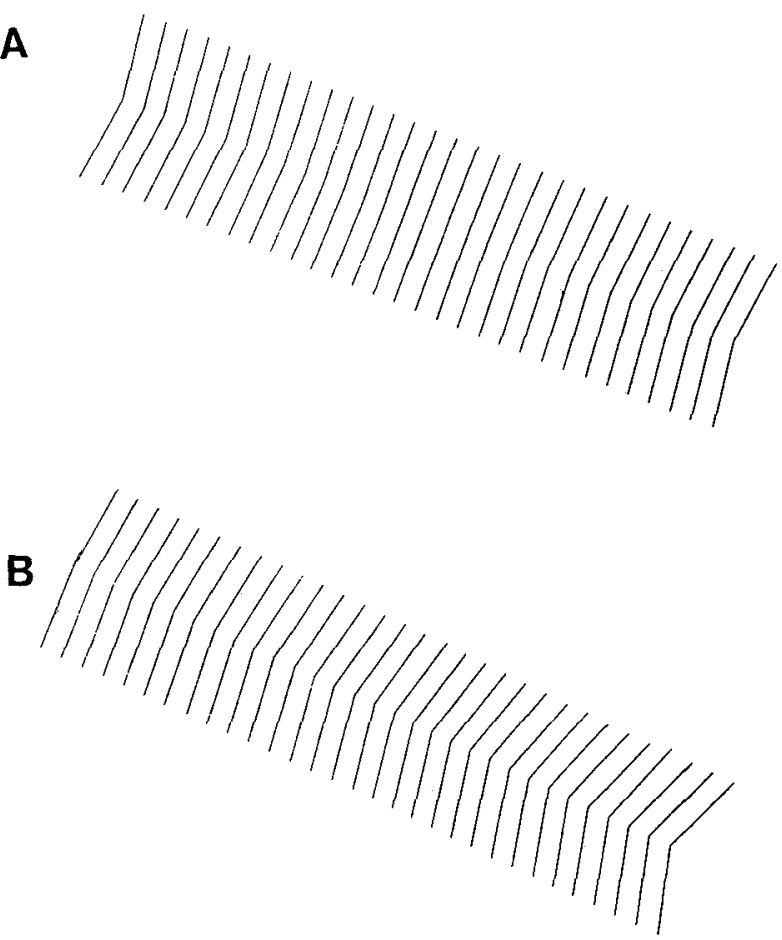

Figure 5. Graded series of matching angles used for (A) Bourdon edges and (B) unbending edges, Experiment 2. The actual series shown to the subject were each on separate sheets, and the angles were lightly numbered in pencil, from left to right, 1-31. Each series was presented at the $22.5^{\circ}$ angle to the horizontal, as shown.

use the Figure 5B series to match the right-hand edge in Figure 4B (or 4D) (unbending pretest); and (4) to use the 5B series to match the right-hand edge in Figure $4 \mathrm{C}$ (unbending test).

The subjects were seated at a low coffee table. The subjective contour figures were placed flat on the table with the graded series directly below the subjective contour figure (between the subject and the figure). The instruction was to look down on the display without tilting the head. Each subject made one judgment of each edge, giving the experimenter a number corresponding to the matching angle, which was later converted to an angular error.

\section{Results}

In Experiment 2.1, with Figure 4B, the mean obtained Bourdon illusion, $-8.1^{\circ}$, was similar to those in Experiment $1\left(-8.08^{\circ}\right.$ and $\left.-7.94^{\circ}\right)$. The Bourdon pretest mean was again slightly positive, a slight unbending effect of $+1.1^{\circ}\left(+0.26^{\circ}\right.$ and $+0.34^{\circ}$ in Experiment 1$)$. In contrast, unbending effects were much larger than in the first experiment: $+6.2^{\circ}$ compared with $+1.88^{\circ}$ and $+1.78^{\circ}$ in Experiment 1. Nevertheless, assuming that the $F$ test is robust to departures from homogeneity of variance, a subjects $\times$ treatments analysis showed that the Bourdon illusion was larger than the unbending effect $[F(1,19)=$ $6.24, p<.025$ ].

In Experiment 2.2, with Figure 4D, the mean obtained Bourdon effect was similar to that in Experiment 2.1 $\left(-7.85^{\circ}\right)$, as was the unbending effect $\left(+6.85^{\circ}\right)$, and the Bourdon pretest mean was also slightly positive again $\left(+1 \cdot 17^{\circ}\right)$. The difference in absolute magnitude between bending and unbending effects was not significant $[F(1,165)=1.31, p>.05]$. However (see below), the variance of the unbending effect in this experiment was more than twice that in Experiment 2.1 and almost 3 times that of the Bourdon effect.

What might explain the larger unbending effects in Experiment 2? Figures 6 and 7 show the frequency distributions of pretest, test, and test minus pretest in Experiments 2.1 and 2.2. First, it can be noted that whereas the Bourdon pretests are clustered fairly tightly around zero, the unbending pretests are much more variable. The variances of the Bourdon and unbending pretests were 2.09 and 17.83, respectively, in Experiment 2.1; they were 3.26 and 21.25 in Experiment 2.2.

This six- to ninefold difference in variance reflects almost all the subjects' observations, made during testing, on the difficulty of the unbending task. The same difference appears in the tests (middle panels) where the Bourdon and unbending variances were 7.80 and 27.23, respectively, in Experiment 2.1 and were 7.63 and 17.33 in Experiment 2.2. Indeed, there is a clear suggestion of a multimodal distribution in the unbending test data, implying that different subjects may have used different criteria of judgment. As a result of these variance differences, the test minus pretest scores (bottom panel) were reasonably clustered for the Bourdon data with almost coincident mode $\left(-8^{\circ}\right)$ and mean $\left(-7.85^{\circ}\right)$ but were far less so for the unbending effects. The variance of the test minus pretest scores was 7.59 for the Bourdon effect and 14.06 for the unbending effect in Experiment 2.1; and the respective variances were 12.24 and 31.83 in Experiment 2.2. In the latter case, there is a clear skew in the unbending data; the mode is at $2^{\circ}$ but the mean is $6.85^{\circ}$. Removing the seven extreme positive scores would reduce the mean to $5.4^{\circ}$.

As in Experiment 1, the unbending pretest mean in Experiment 2 clearly showed a bending effect. The mean matching error in Experiment 2.1 was $-2.85^{\circ}$, significantly different from zero even using its own large standard error $[t(19)=-3.03, p<.01]$. In Experiment 2.2, this mean was $-3.52^{\circ}[t(33)=-4.46, p<.0005]$.

Figure 8 shows the mean pretest and test Bourdon and unbending effects and standard errors, together with the Bourdon pretest and test means from Experiment 1. This figure highlights (1) the similarity of the Bourdon data in all of the experiments, (2) the variability in the unbending data of Experiment 2, (3) the similarity of the Experiment 2.1 and 2.2 results, and (4) the relatively large contribution of the pretest bending effect to the overall unbending effect in Experiment 2. Clearly, the positioning of the pretest chevron within the circular contours (Figures 4B and 4C) had no significant effect on the unbending pretest mean.

It is important to note that, although the absolute test minus pretest unbending and Bourdon means did not differ significantly in Experiment 2.2 because, we claim, the variances were so large, nevertheless the unbending and 

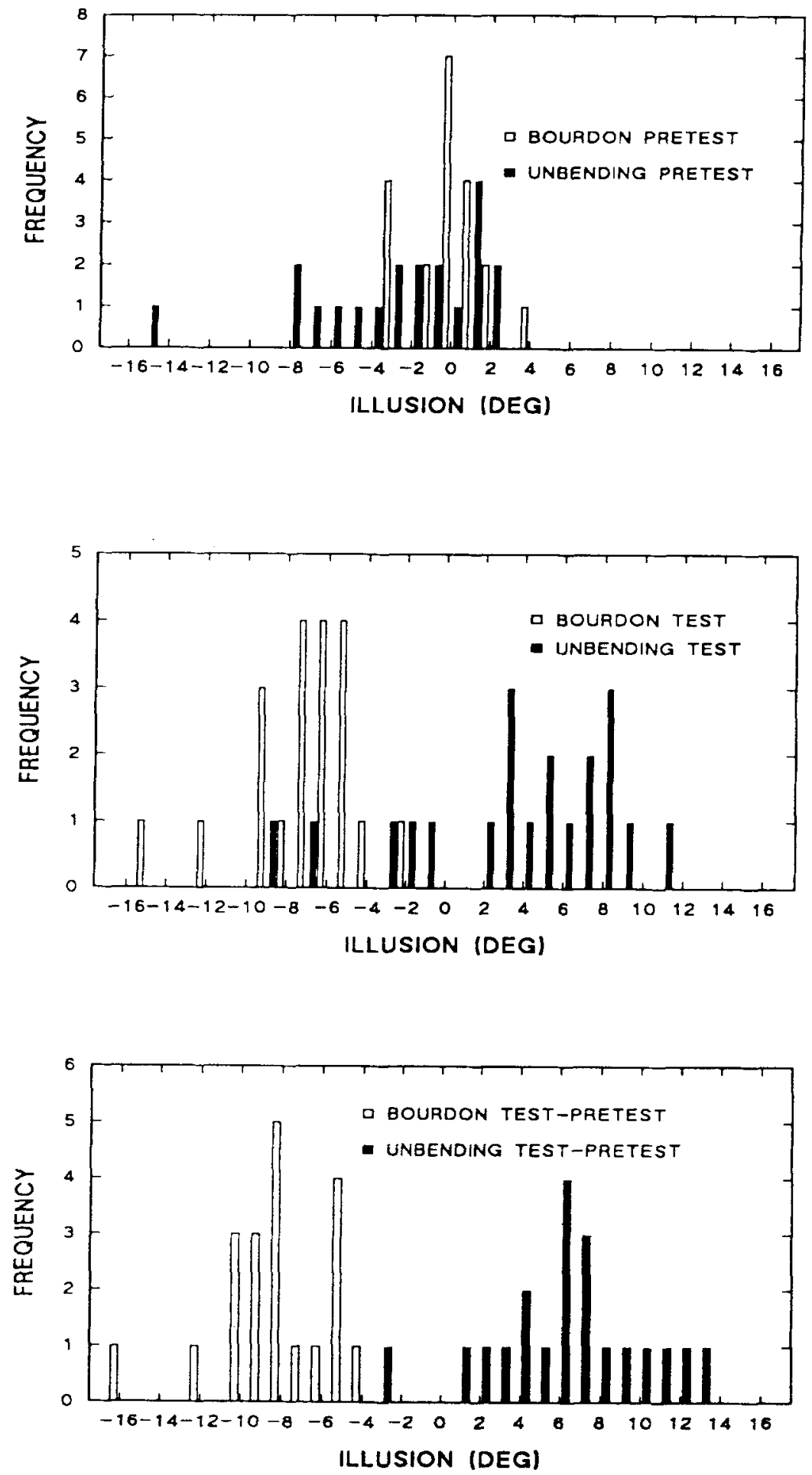

Figure 6. Frequency distributions of Bourdon and unbending angular matching errors in pretest and test conditions (upper and middle panels) and the test minus pretest scores (bottom panel), Experiment 2.1. 

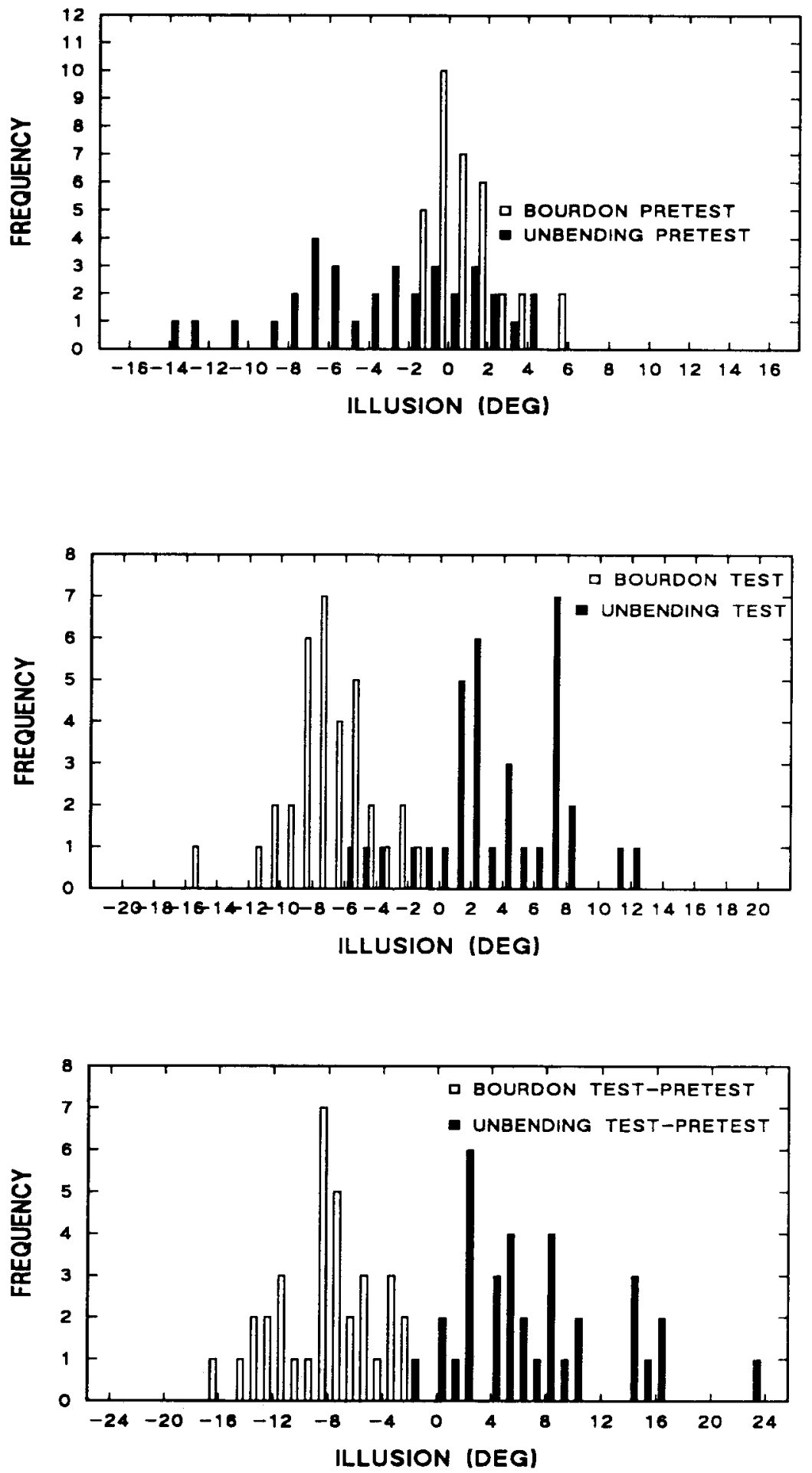

Figure 7. As for Figure 6, Experiment 2.2. 


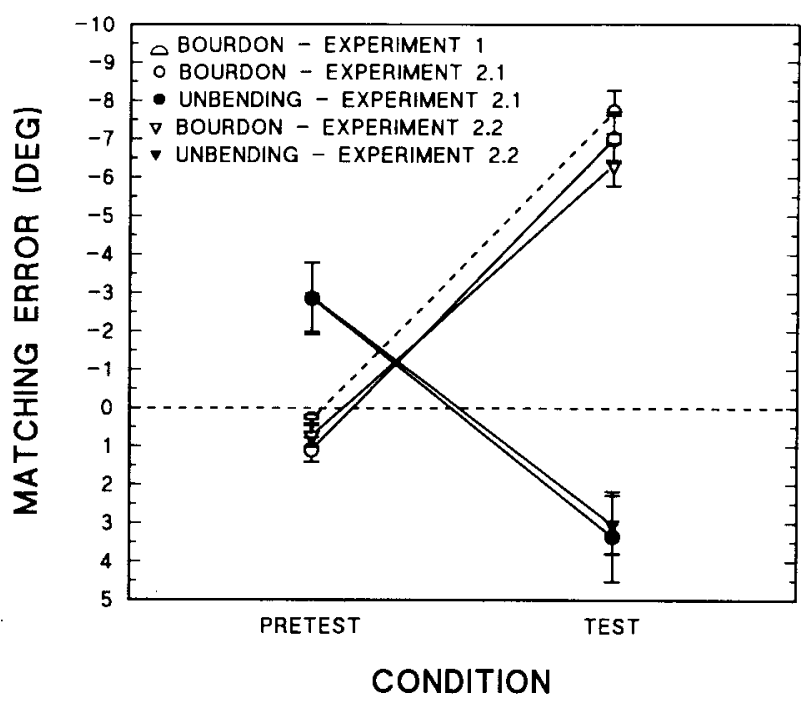

Figure 8. Mean angular matching errors for pretests and tests, Experiment 2. Semicircular symbols show Bourdon illusions from Experiment 1. Error bars show standard errors.

Bourdon pretest means were different $[F(1,165)=23.51$, $p<.0005]$, as were the absolute test means $[F(1,165)$ $=11.91, p<.001]$.

\section{GENERAL DISCUSSION}

The data of Experiments 1 and 2 not only falsify the theories put forward by Walker and Shank (1988b) to explain Bourdon and unbending effects but also suggest why unbending effects are smaller when the chevron matching technique is used and why unbending effects are smaller with real rather than subjective contours.

Walker and Shank (1988b) proposed two models, a feature model and a confusion model, both of which predict, as they acknowledge, equally sized bending and unbending effects. Although their data from the use of subjective (but not objective) contours were consistent with these models, the data of the present experiments are not: In both Experiment 1 and 2.1, Bourdon effects were significantly greater than unbending effects; and in Experiment 2.2, the Bourdon test mean exceeded the unbending test mean significantly. The nonsignificant difference in that experiment between the pretest corrected measures was due not only to the large and significant bending effect in the pretest but also to the large skew in the test minus pretest data. The previous findings of Wenderoth et al. (1986a), discussed in the introduction, coupled with the evidence presented here for the clear inequality of bending and unbending effects, must seriously call into question the Walker and Shank theories. We therefore reject those theories.

Wenderoth and O'Connor (1987a, 1987b) and Wenderoth et al. (1986a) proposed that the Bourdon illusion represents a compromise between separate neural channels coding orientation, position, and collinearity. Part of their evidence for this view arose from informal ob- servations that the subjects, in performing the matching task, first adjusted the chevron arms so that they appeared parallel to the test arms and then made an additional bending adjustment following global inspection of the figures. It was as if a local orientation match was not sufficient, and subsequent global inspection revealed an extra illusion. Wenderoth and O'Connor (1987b) reported that no such second-stage adjustment occurred with truly bent edges.

Wenderoth (1990) has proposed that this second-stage adjustment reflects the position effect that can be seen in Figure 1A; even were the upper and lower chevron segments set parallel to the adjacent wedge edges, more global inspection of the whole Bourdon figure gives the clear impression that the left-hand upper and lower extremities (i.e., the left edges of the wedge bases) are displaced to the right of the central apices. Thus, in Figure $1 \mathrm{~A}$, attraction orientation effects and position effects occur in the same direction and reinforce each other. Since acuity for nonstraightness or noncollinearity is high, a large effect occurs. In contrast, in Figure 1B, orientation and position effects are directionally opposite: The left edge of the upper wedge appears to be oriented clockwise more than it actually is, but the wedge base edges appear to be displaced in position to the left of the wedge apices. Not only will these directionally opposite effects reduce the overall illusion, but, as Wenderoth and O'Connor (1987b) suggested on the basis of six experiments, secondstage adjustments may only occur where acuity for departures from the property being judged is high (e.g., collinearity, verticality, parallelism, etc.) and may not occur otherwise, especially when no internal standard exists for comparison (e.g., departure from an angle of $155^{\circ}$, as in the unbending effect).

In Experiment 1, then, subjects making parallel or chevron matches to the truly straight Bourdon edge would have made a second-stage adjustment when the initial parallel setting (near straight) looked insufficiently bent due to the position effect and high acuity for apparent noncollinearity. Unbending adjustments would not have had this second-stage adjustment, partly because orientation and position effects are directionally opposite and partly because acuity for departure from a $155^{\circ}$ angle is low. Also, the task does not in itself require a judgment of orientation or angular subtense. That is, a parallel match can be made without judging absolute orientation or angular subtense. Indeed, parallel matches to angle arms do not predict angular matches (Wenderoth \& White, 1979). In Experiment 2, subjects were forced to judge angles by the nature of the task, and so the larger but much more variable unbending effects in that experiment may reflect quite different mechanisms as well as more complex interactions than the effects in Experiment 1. Further investigation is clearly needed to establish why chevron matching and angle matching give similar Bourdon illusions but such different unbending effects, with much larger but more variable effects using the latter method.

We now turn to the general question of why some illusions are larger in subjective than in real contour displays, 
and particularly to the question of why unbending effects are larger in subjective contours. Recently, Paradiso et al. (1989) measured tilt aftereffects using various combinations of real and subjective inducing and test stimuli. Their data, especially those from the second experiment, suggest that aftereffects are larger when all contours are subjective than when all are real. Over 3 observers, the mean effects were $2.49^{\circ}$ and $1.08^{\circ}$ for subjective and real contours, respectively. Paradiso et al. also found, as Walker and Shank (1988a) have reported with the Bourdon effect, that real inducing stimuli affect subjective test stimuli more than subjective inducing stimuli affect real test stimuli. The first of these results-that all-subjective contour effects are larger - is consistent with our hypothesis (above) that our pretest bending effects were due to larger obtuse angle contraction in the subjective test figure than in the real matching chevron. Both findings suggest why Bourdon and tilt illusions are larger in subjective than in real contours. Vogels and Orban (1987) reported that the JND for orientation discrimination is greater using subjective rather than real contours. When the JND is greater, the tuning curve is broader, and both mean effects and their variances should be larger. Carpenter and Blakemore (1973) demonstrated this when they showed that tilt illusions are larger when the test line is oblique and the inducing line is vertical, as compared with the opposite arrangement. In the data of Paradiso et al. (1989), the average standard error of the larger subjective contour aftereffects $(0.43)$ was almost double that of the real contour effects $(0.25)$. Thus, we suggest that tilt illusions and aftereffects are larger when subjective contours are used because the JND for orientation discrimination is larger than it is with real contours.

But consider the angle problem. Why is the unbending effect larger with subjective than with real contours? In both our experiments, we found a bending effect in the pretest control measure. This we attribute to the fact that obtuse angle contraction effects (tilt illusions) are larger in the judged subjective contour than in the real matching contour, whether the real matching contour is a chevron (Experiment 1) or an angle (Experiment 2). With real unbending contours, there would be no such bending error in the pretest; the solid pretest symbol in Figure 8 would lie on the dotted zero line and the unbending effect would be reduced, in this case from $6.2^{\circ}$ to $3.35^{\circ}$ in Experiment 2.1 and from $6.85^{\circ}$ to $3.32^{\circ}$ in Experiment 2.2. (It should be noted that Walker and Shank (1987, 1988a, 1988b) never used pretests, so that Experiments 2.1 and 2.2 , in their terms, gave unbending test means of $3.35^{\circ}$ and $3.32^{\circ}$, only $41 \%$ and $48 \%$ of the $8.1^{\circ}$ and $7.85^{\circ}$ Bourdon effects, respectively.) Finally, given the variability of the unbending data in Experiment 2, and the difficulty of the unbending judgments acknowledged by all subjects, we doubt the validity of the angle-matching task as a measure of unbending effects, even though we sought to remove any bias that may have existed in Walker and Shank's graded series. Perhaps something in their method reduced variability (which they do not generally report), such as subjects' tending to select one of the displayed anchor stimuli rather than interpolating. It would be of interest to see frequency distributions of their test data.

\section{REFERENCES}

Carpenter, R. H. S., \& Blakemore, C. (1973). Interactions between orientations in human vision. Experimental Brain Research, 18, 287-303.

Harris, J. P., \& Calvert, J. E. (1985). The tilt after-effect: Changes with stimulus size and eccentricity. Spatial Vision, 1, 113-129.

HowaRD, I. P. (1982). Human visual orientation. New York: Wiley.

O'ToOle, B., \& Wenderoth, P. (1977). The tilt illusion: Repulsion and attraction effects in the oblique meridian. Vision Research, 17, 367-374.

Paradiso, M. A., Shimojo, S., \& Nakayama, K. (1989). Subjective contours, tilt aftereffects, and visual cortical organization. Vision Research, 29, 1205-1213.

Rozvany, G. I. N., \& Day, R. H. (1980). Determinants of the Bourdon effect. Perception \& Psychophysics, 28, 39-44.

Vogels, R., \& OrbaN, G. A. (1987). Illusory contour orientation determination. Vision Research, 27, 453-467.

von der Heydt, R., \& Peterhans, S. (1989). Mechanisms of contour perception in monkey visual cortex: 1. Lines of Pattern discontinuity. Soumal of Neuroscience, 9, 1731-1748.

Walker, J. T., \& Shank, M. D. (1987). The Bourdon illusion in subjective contours. Perception \& Psychophysics, 42, 15-24.

WALKER, J. T., \& SHANK, M. D. (1988a). Interactions between real and subjective contours in the Bourdon illusion. Perception \& Psychophysics, 43, 567-574.

Walker J. T., \& Shank, M. D. (1988b). Real and subjective lines and edges in the Bourdon illusion. Perception \& Psychophysics, 43, $475-484$.

Wallace, G. K., \& Crampin, D. J. (1969). The effect of background density on the Zöllner illusion. Vision Research, 9, 167-177.

WENDEROTH, P. (1990). The basis of the Bourdon illusion. Manuscript submitted for publication.

Wenderoth, P., Johnstone, S. (1987). Possible neural substrates for orientation analysis and perception. Perception, 16, 693-709.

WeNDEROTH, P., \& O'CONNOR, T. (1987a). The effects of display and observer strategy variables on Bourdon assimilation illusions. Perception, 16, 79-88.

Wenderoth, P., \& O'Connor, T. (1987b). Outline- and solid-angle orientation illusions have different determinants. Perception \& Psychophysics, 41, 45-52.

Wenderoth, P., O'Connor, T., Johnson, M. (1986a). Expansion and contraction of outline and solid acute angles: Effects of angle magnitude, type of display, and the nature of the matching task. Perception \& Psychophysics, 39, 261-266.

Wenderoth, P., O'Connor, T., Johnson, M. (1986b). The tilt illusion as a function of the relative and absolute lengths of test and inducing lines. Perception \& Psychophysics, 39, 339-345.

Wenderoth, P., \& White, D. (1979). Angle-matching illusions and perceived line orientation. Perception, 8, 565-575.

(Manuscript received November 6, 1989; revision accepted for publication July 6, 1990.) 